Canad. Math. Bull. Vol. 23 (3), 1980

\title{
SOME TRIANGLE INEQUALITIES AND GENERALIZATIONS
}

\author{
BY
}

C. E. CARROLL, C. C. YANG AND S. AHN

\begin{abstract}
Let $D_{n, s}(x)=\Pi\left(-s a_{1}^{x}+a_{2}^{x}+\cdots+a_{n}^{x}\right)$, where $a_{i}$, $s$, and $x$ are real, and $\Pi$ denotes the product over cyclic rearrangements of the subscripts. We show that, in five special cases, $D_{n, s}(x) D_{n, s}(y)$ is greater than a fixed multiple of $D_{n, s}(x+y)$.
\end{abstract}

Introduction and results. A plane triangle $A B C$ has an incircle of radius $r$ and a circumcircle of radius $R$. By a known trigonometric formula [5, p. 200],

$$
2 r^{2}-4 R^{2} \cos A \cos B \cos C=I P^{2} \geq 0 .
$$

In terms of the sides of the triangle, we obtain

(1) $(-a+b+c)^{2}(a-b+c)^{2}(a+b-c)^{2}$

$$
\geq\left(-a^{2}+b^{2}+c^{2}\right)\left(a^{2}-b^{2}+c^{2}\right)\left(a^{2}+b^{2}-c^{2}\right) .
$$

The functions appearing here are special cases of the general function

$$
D_{n, s}(x)=\Pi\left(-s a_{1}^{x}+a_{2}^{x}+a_{3}^{x}+\cdots+a_{n}^{x}\right),
$$

where $s$ and $x$ are real and $\Pi$ denotes the product over the $n$ cyclic rearrangements of the subscripts. Although (1) holds for all real $a, b$, and $c$, our theorems $1,2,3$, and 5 are valid only for positive values of $a_{1}, a_{2}, \ldots, a_{n}$. The inequalities connecting $D_{n, s}(x) D_{n, s}(y)$ and $D_{n, s}(x+y)$ seem not to appear in standard treatises on inequalities $[1,4,7]$, but we shall mention a slight connection between the work of Gårding [3] and the proof of

Theorem 1. If $n>1$ and $s \leq 0$, then $D_{n, s}(x) D_{n, s}(y)>(-s) D_{n, s}(x+y)$.

The inequalities for positive values of $s$ are more interesting and more difficult.

THEOREM 2.

$$
\left[D_{4,1}(1)\right]^{2}>3 D_{4,1}(2) \text {. }
$$

We remark that (2) becomes an equality if we set $a_{1}=a_{2}=a_{3}$ and take the limit as $a_{4} \rightarrow 0$. If we let $a_{4} \rightarrow 0$ and then use the elementary inequality $3\left(a^{2}+b^{2}+\right.$ $\left.c^{2}\right) \geq(a+b+c)^{2}$, we recover (1).

Received by the editors September 30, 1977 and, in revised form, February 20, 1979. 
Jensen's inequality [6] suggests the following generalizations:

THEOREM 3. If $x$ and $y$ are positive, then

$$
D_{3,1}(x) D_{3,1}(y) \geq D_{3,1}(x+y),
$$

with equality iff $a_{1}=a_{2}=a_{3}$.

Theorem 4. If $a_{1}, a_{2}, s, x$, and $y$ are positive, or if $s>0$ and $x$ and $y$ are positive integers,

$$
D_{2, s}(x) D_{2, s}(y) \geq(1-s)^{2} D_{2, s}(x+y),
$$

with equality only if $\left|a_{1}\right|=\left|a_{2}\right|$.

Finally, we shall prove

Theorem 5. Suppose $a_{1}^{x+y}, a_{2}^{x+y}, a_{3}^{x+y}$ are the sides of a triangle. If $x$ and $y$ are positive and $s \leq 1$, then

$$
D_{3, s}(x) D_{3, s}(y)>2(1-s)^{2} D_{3, s}(x+y) .
$$

Proof of Theorem 1. The proof is trivial if $s=0$. If $n>1$ and $s<0$, we have $D_{n, s}(x) D_{n, s}(y)>\Pi\left(s^{2} a_{1}^{x+y}+a_{2}^{x+y}+\cdots+a_{n}^{x+y}\right)$. The quantity on the right is greater than $(-s) D_{n, s}(x+y)$, except that it is equal to $D_{n, s}(x+y)$ when $s=-1$. To prove this, we need

Lemma 1. If $A_{1}, A_{2}, \ldots, A_{n}$ are positive, $n>1, s<0$, and $s \neq-1$, then

$$
\Pi\left(s^{2} A_{1}+A_{2}+\cdots+A_{n}\right)+s \Pi\left(-s A_{1}+A_{2}+\cdots+A_{n}\right)
$$

is positive.

The proof can be done by induction on $n$ or by use of Cauchy's inequality followed by Hölder's inequality. We plan to give full details and extensions of Lemma 1 in a separate publication. We conjecture that the polynomial (6) is hyperbolic in the sense of Gårding [3].

Proof of Theorem 2. If the numbers $a_{1} \cdots a_{4}$ are not all distinct, the inequality is easily proved. Let $a_{1}=a_{2}$. Then

$$
D_{4,1}(1)=4 a_{2}^{2}\left(a_{3}+a_{4}\right)^{2}-\left(a_{3}^{2}-a_{4}^{2}\right)^{2}
$$

and

$$
D_{4,1}(2)=4 a_{2}^{4}\left(a_{3}^{2}+a_{4}^{2}\right)^{2}-\left(a_{3}^{2}-a_{4}^{2}\right)^{2}\left(a_{3}^{2}+a_{4}^{2}\right)^{2} \text {. }
$$

Hence,

$$
12 a_{2}^{4}\left(a_{3}+a_{4}\right)^{4}-3\left(a_{3}^{2}-a_{4}^{2}\right)^{4}>3 D_{4,1}(2) .
$$

Finally,

$$
\left[D_{4,1}(1)\right]^{2}-12 a_{2}^{4}\left(a_{3}+a_{4}\right)^{4}+3\left(a_{3}^{2}-a_{4}^{2}\right)^{4}=\left[2 a_{2}^{2}\left(a_{3}+a_{4}\right)^{2}-2\left(a_{3}^{2}-a_{4}^{2}\right)^{2}\right]^{2} \geq 0 .
$$


In the general case, we can write $D_{4,1}(1)$ and $D_{4,1}(2)$ in terms of the elementary symmetric functions $\sigma_{1} \cdots \sigma_{4}$. They are defined by

$$
\Pi\left(x-a_{i}\right) \equiv x^{4}-\sigma_{1} x^{3}+\sigma_{2} x^{2}-\sigma_{3} x+\sigma_{4} .
$$

Some computations give

$$
\begin{aligned}
D_{4,1}(1)= & -\sigma_{1}^{4}+4 \sigma_{1}^{2} \sigma_{2}-8 \sigma_{1} \sigma_{3}+16 \sigma_{4}, \\
D_{4,1}(2)= & -\left(\sigma_{1}^{2}-2 \sigma_{2}\right)^{4}+4\left(\sigma_{1}^{2}-2 \sigma_{2}\right)^{2}\left(\sigma_{2}^{2}-2 \sigma_{1} \sigma_{3}+2 \sigma_{4}\right) \\
& -8\left(\sigma_{1}^{2}-2 \sigma_{2}\right)\left(\sigma_{3}^{2}-2 \sigma_{2} \sigma_{4}\right)+16 \sigma_{4}^{2},
\end{aligned}
$$

and

$$
\left[D_{4,1}(1)\right]^{2}-3 D_{4,1}(2)=P\left(\sigma_{1}, \sigma_{2}, \sigma_{3}\right)-8\left(7 \sigma_{1}^{4}-22 \sigma_{1}^{2} \sigma_{2}+32 \sigma_{1} \sigma_{3}-26 \sigma_{4}\right) \sigma_{4},
$$

where $P\left(\sigma_{1}, \sigma_{2}, \sigma_{3}\right)$ is a polynomial. We shall show that (8) is a decreasing function of $\sigma_{4}$, when $\sigma_{1}, \sigma_{2}, \sigma_{3}$ are fixed. Then, if (8) is positive at the largest allowed value of $\sigma_{4}$, it is always positive. If (7) has distinct real zeroes, its value at each local minimum must be negative. We may increase $\sigma_{4}$ until the value at one of the local minima is zero; then two zeroes coincide and we have the case first considered. Thus, (8) is always positive.

To show that (8) is a decreasing function of $\sigma_{4}$, we need inequalities relating the symmetric functions. We shall use the method of Breusch [2] to show that

$$
13 \sigma_{1}^{3}-44 \sigma_{1} \sigma_{2}+64 \sigma_{3}>0 \text {. }
$$

By the arithmetic-geometric inequality, $\sigma_{1}^{4} \geq 4^{4} \sigma_{4}>104 \sigma_{4}$. Combining this with (9) gives $7 \sigma_{1}^{4}-22 \sigma_{1}^{2} \sigma_{2}+32 \sigma_{1} \sigma_{3}-52 \sigma_{4}>0$, showing that (8) is a decreasing function of $\sigma_{4}$.

Our last step is to prove (9). Since the left side is a homogeneous polynomial, we can set $\sigma_{1}=1$ without loss of generality. Since $\sigma_{3}$ is positive, (9) holds when $\sigma_{2}<\frac{1}{4}$. Hence, we assume $\sigma_{2} \geq \frac{1}{4}$. The polynomial (7) has four real zeroes. Its derivative,

$$
4\left[\left(x-\frac{1}{4}\right)^{3}+\left(\frac{1}{2} \sigma_{2}-\frac{3}{16}\right)\left(x-\frac{1}{4}\right)+\left(\frac{1}{8} \sigma_{2}-\frac{1}{4} \sigma_{3}-\frac{1}{32}\right)\right],
$$

must have three real zeroes. We compute the discriminant of this cubic polynomial, as suggested by Breusch [2], obtaining

$$
32\left(\sigma_{2}-\frac{3}{8}\right)^{3}+27\left(\sigma_{2}-2 \sigma_{3}-\frac{1}{4}\right)^{2} \leq 0 .
$$

But the quantity on the left is positive, unless $\sigma_{2} \leq \frac{3}{8}$. This bound for $\sigma_{2}$ could be obtained more easily from Maclaurin's inequality [1, 4]. From (10), we have

$$
13-44 \sigma_{2}+64 \sigma_{3} \geq 5-12 \sigma_{2}-8\left(1-\frac{8}{3} \sigma_{2}\right)^{3 / 2} \text {. }
$$

We now minimize the function on the right side, using the bounds for $\sigma_{2}$. Since this function has a negative second derivative at interior points of the interval $\left[\frac{1}{4}, \frac{3}{8}\right]$, the minimum is at one of the end points. The values at both end points are positive, which completes the proof. 
Proof of Theorem 3. We shall prove (3) and

$$
D_{3,1}(x) D_{3,1}(y) \leq\left[D_{3,1}\left(\frac{x+y}{2}\right)\right]^{2}
$$

by use of Jensen's inequality [6]. Most of the relevant properties of $D_{3,1}(x)$ are stated in two lemmas.

Lemma 2. If $D_{3,1}(x) \neq 0$, then

$$
\frac{d^{2}}{d x^{2}} \log \left|D_{3,1}(x)\right| \leq 0
$$

with equality iff $a_{1}=a_{2}=a_{3}$.

Proof. We may assume $a_{1} \geq a_{2} \geq a_{3}$ without loss of generality. Since $D_{3,1}(x) \neq 0$, no vanishing denominators appear in

$$
\begin{aligned}
\frac{d^{2}}{d x^{2}} \log \left|D_{3,1}(x)\right|= & \frac{-a_{1}^{x} a_{2}^{x}\left(\log \frac{a_{1}}{a_{2}}\right)^{2}+a_{2}^{x} a_{3}^{x}\left(\log \frac{a_{2}}{a_{3}}\right)^{2}-a_{3}^{x} a_{1}^{x}\left(\log \frac{a_{3}}{a_{1}}\right)^{2}}{\left(-a_{1}^{x}+a_{2}^{x}+a_{3}^{x}\right)^{2}} \\
& +\frac{-a_{1}^{x} a_{2}^{x}\left(\log \frac{a_{1}}{a_{2}}\right)^{2}-a_{2}^{x} a_{3}^{x}\left(\log \frac{a_{2}}{a_{3}}\right)^{2}+a_{3}^{x} a_{1}^{x}\left(\log \frac{a_{3}}{a_{1}}\right)^{2}}{\left(-a_{1}^{x}+a_{2}^{x}+a_{3}^{x}\right)^{2}} \\
& +\frac{a_{1}^{x} a_{2}^{x}\left(\log \frac{a_{1}}{a_{2}}\right)^{2}-a_{2}^{x} a_{3}^{x}\left(\log \frac{a_{2}}{a_{3}}\right)^{2}-a_{3}^{x} a_{1}^{x}\left(\log \frac{a_{3}}{a_{1}}\right)^{2}}{\left(a_{1}^{x}+a_{2}^{x}-a_{3}^{x}\right)^{2}}
\end{aligned}
$$

The first numerator is never positive, and $\left(-a_{1}^{x}+a_{2}^{x}+a_{3}^{x}\right)^{-2} \geq\left(a_{1}^{x}-a_{2}^{x}+a_{3}^{x}\right)^{-2}$. Hence, $-2 a_{1}^{x} a_{2}^{x}\left(\log \frac{a_{1}}{a_{2}}\right)^{2}\left(a_{1}^{x}-a_{2}^{x}+a_{3}^{x}\right)^{-2}$ is an upper bound for the first two terms on the right side of (13). Further estimation gives

$$
\frac{d^{2}}{d x^{2}} \log \left|D_{3,1}(x)\right| \leq \frac{-a_{1}^{x} a_{2}^{x}\left(\log \frac{a_{1}}{a_{2}}\right)^{2}-a_{2}^{x} a_{3}^{x}\left(\log \frac{a_{2}}{a_{3}}\right)^{2}-a_{3}^{x} a_{1}^{x}\left(\log \frac{a_{3}}{a_{1}}\right)^{2}}{\left(a_{1}^{x}+a_{2}^{x}-a_{3}^{x}\right)^{2}}
$$

which is negative unless $a_{1}=a_{2}=a_{3}$. We note that (12) is also valid when $x \leq 0$.

The uniqueness of the positive real zero of $D_{3,1}(x)$ is essential for the proof of Theorem 3. This uniqueness follows from

Lemma 3. $D_{3,1}(x) /\left(a_{1} a_{2} a_{3}\right)^{x}$ is a monotonic decreasing function of $x$, unless $a_{1}=a_{2}=a_{3}$.

Proof. If $x$ is less than the first positive zero of $D_{3,1}(x)$, we note that $\frac{d}{d x} \log \frac{D_{3,1}(x)}{\left(a_{1} a_{2} a_{3}\right)^{x}}$ vanishes at $x=0$, and use Lemma 2 to show that $\log \frac{D_{3,1}(x)}{\left(a_{1} a_{2} a_{3}\right)^{x}}$ 
is decreasing, unless $a_{1}=a_{2}=a_{3}$. If $D_{3,1}(x) \leq 0$, we may assume $a_{1}>a_{2} \geq a_{3}$ without loss of generality. Then

$$
-\frac{D_{3,1}(x)}{\left(a_{1} a_{2} a_{3}\right)^{x}}=\left[1-\left(\frac{a_{2}}{a_{1}}\right)^{x}-\left(\frac{a_{3}}{a_{1}}\right)^{x}\right]\left[\left(\frac{a_{1}}{a_{2}}\right)^{x}+1-\left(\frac{a_{3}}{a_{2}}\right)^{x}\right]\left[\left(\frac{a_{1}}{a_{3}}\right)^{x}-\left(\frac{a_{2}}{a_{3}}\right)^{x}+1\right]
$$

is the product of three increasing functions; the first factor is non-negative and the other two are positive.

We can now prove (11). If $D_{3,1}\left(\frac{x+y}{2}\right)=0$ and $x \neq y$, then Lemma 3 implies $D_{3,1}(x) D_{3,1}(y)<0$. If $D_{3,1}\left(\frac{x+y}{2}\right) \neq 0$, then either $D_{3,1}(x)$ or $D_{3,1}(y)$ has the same sign as $D_{3,1}\left(\frac{x+y}{2}\right)$. If all three of them have the same sign, we obtain (11) from Lemma 2 and Jensen's inequality; if not, (11) still holds.

Theorem 3 is easily verified if $D_{3,1}(x) D_{3,1}(y) D_{3,1}(x+y)=0$. If $D_{3,1}(x+y)>$ 0 , then $D_{3,1}(x)$ and $D_{3,1}(y)$ are positive. Using Lemma 2 and Jensen's inequality, we find

(14) $\log D_{3,1}(x) \geq \frac{x}{x+y} \log D_{3,1}(x+y)+\frac{y}{x+y} \log D_{3,1}(0)=\frac{x}{x+y} \log D_{3,1}(x+y)$ and, similarly,

$$
\log D_{3,1}(y) \geq \frac{y}{x+y} \log D_{3,1}(x+y) .
$$

In both cases, equality holds iff $a_{1}=a_{2}=a_{3}$. Addition of (14) and (15) gives (3). If $D_{3,1}(x+y)<0$, the proof is trivial unless $D_{3,1}(x)$ and $D_{3,1}(y)$ have opposite signs. we assume $D_{3,1}(x)<0<D_{3,1}(y)$, and use Lemma 3 to show

and

$$
\frac{\left|D_{3,1}(x)\right|}{\left(a_{1} a_{2} a_{3}\right)^{x}}<\frac{\left|D_{3,1}(x+y)\right|}{\left(a_{1} a_{2} a_{3}\right)^{x+y}}
$$

$$
\frac{D_{3,1}(y)}{\left(a_{1} a_{2} a_{3}\right)^{y}}<D_{3,1}(0)=1 \text {. }
$$

Therefore, $\left|D_{3,1}(x)\right| D_{3,1}(y)<\left|D_{3,1}(x+y)\right|$, which completes the proof.

Proof of Theorem 4. If $a_{1}$ and $a_{2}$ are positive, then $D_{2, s}(0)=(1-s)^{2}$,

$$
\frac{D_{2, s}(x)}{\left(a_{1} a_{2}\right)^{x}}=1+s^{2}-s\left[\left(\frac{a_{1}}{a_{2}}\right)^{x}+\left(\frac{a_{2}}{a_{1}}\right)^{x}\right]
$$

is a non-increasing function of $x$, and the proof of (4) is similar to that of (3). If $a_{1} a_{2} \geq 0$, or if $a_{1}+a_{2}=0$, (4) is easily verified. Thus, we assume $a_{1} a_{2}<0$ and $a_{1}+a_{2} \neq 0$. If $x$ is an odd integer, then $D_{2, s}(x)<0$ and

$$
\frac{\left|D_{2, s}(x)\right|}{\left|a_{1} a_{2}\right|^{x}}=1+s^{2}+s\left(\left|\frac{a_{1}}{a_{2}}\right|^{x}+\left|\frac{a_{2}}{a_{1}}\right|^{x}\right)
$$


is an increasing function of $x$. If $y$ is even, this gives

and we also have

$$
\frac{\left|D_{2, s}(x)\right|}{\left|a_{1} a_{2}\right|^{x}}<\frac{\left|D_{2, s}(x+y)\right|}{\left|a_{1} a_{2}\right|^{x+y}},
$$

$$
\frac{D_{2, s}(y)}{\left(a_{1} a_{2}\right)^{y}}<D_{2, s}(0)=(1-s)^{2} .
$$

Hence, $\left|D_{2, s}(x)\right| D_{2, s}(y)<(1-s)^{2}\left|D_{2, s}(x+y)\right|$. In this way, (4) is proved when $x$ and $y$ are integers of opposite parity. If $x$ and $y$ are both odd, $D_{2, s}(x+y)$ is unchanged when we replace $a_{1}$ and $a_{2}$ by their absolute values, while $D_{2, s}(x) D_{2, s}(y)$ decreases, so (4) must hold. Finally, (4) holds when $x$ and $y$ are both even.

Proof of Theorem 5. Since $a_{1}^{x+y}, a_{2}^{x+y}, a_{3}^{x+y}$ form a triangle, $D_{3,1}(x+y)$ is positive; but (5) remains valid if it vanishes. To prove this, we shall use

$$
0 \leq D_{3,1}(x+y) \leq\left(a_{1} a_{2} a_{3}\right)^{x+y} .
$$

To prove the second half of this inequality, let $x+y=1$; then

$$
\begin{aligned}
2 a_{1} a_{2} a_{3}-2 D_{3,1}(1)= & \left(-a_{1}+a_{2}+a_{3}\right)\left(a_{2}-a_{3}\right)^{2}+\left(a_{1}-a_{2}+a_{3}\right)\left(a_{1}-a_{3}\right)^{2} \\
& +\left(a_{1}+a_{2}-a_{3}\right)\left(a_{1}-a_{2}\right)^{2}
\end{aligned}
$$

is non-negative.

The inequality published by Jensen [6] and Pringsheim [8], and attributed to Lüroth, is useful. It implies that both triples $\left(a_{1}^{x}, a_{2}^{x}, a_{3}^{x}\right)$ and $\left(a_{1}^{y}, a_{2}^{y}, a_{3}^{y}\right)$ satisfy triangle inequalities. Hence,

$$
D_{3,1}(x)>0 \quad \text { and } \quad D_{3,1}(y)>0,
$$

and (5) holds when $s=1$.

If $s \leq 0$, we note that the sign of the inequality in Lemma 2 is reversed. Jensen's inequality gives $D_{3, s}(x) D_{3, s}(y) \geq\left[D_{3, s}\left(\frac{x+y}{2}\right)\right]^{2}$. To prove (5), we shall show that

$$
\left[D_{3, s}\left(\frac{x+y}{2}\right)\right]^{2}>2(1-s)^{2} D_{3, s}(x+y) \text {. }
$$

As in the previous paragraph, we have $D_{3, s}\left(\frac{x+y}{2}\right)>0$. We set $x+y=2$, without loss of generality. Then

and

$$
D_{3, s}(1)=-s \sigma_{1}^{3}+(s+1)^{2} \sigma_{1} \sigma_{2}-(s+1)^{3} \sigma_{3}
$$

$$
D_{3, s}(2)=-s\left(\sigma_{1}^{2}-2 \sigma_{2}\right)^{3}+(s+1)^{2}\left(\sigma_{1}^{2} \sigma_{2}^{2}-2 \sigma_{2}^{3} \sigma_{3}+4 \sigma_{1} \sigma_{2} \sigma_{3}\right)-(s+1)^{3} \sigma_{3}^{2},
$$

where $\sigma_{1}, \sigma_{2}, \sigma_{3}$ are the elementary symmetric functions of $a_{1}, a_{2}, a_{3}$. We shall use $s \leq 0$ and

$$
D_{3,1}(1)=4 \sigma_{1} \sigma_{2}-\sigma_{1}^{3}-8 \sigma_{3}>0
$$


to show that

$\left[D_{3, s}(1)\right]^{2}-2(1-s)^{2} D_{3, s}(2)$

$$
\begin{aligned}
= & {\left[(s-1)^{4} \sigma_{2}^{2}+s^{2} \sigma_{1}^{2} \sigma_{2}-s\left(2 s^{2}-3 s+2\right) \sigma_{1}^{2}\left(\sigma_{1}^{2}-3 \sigma_{2}\right)\right]\left(4 \sigma_{2}-\sigma_{1}^{2}\right) } \\
& +2(s+1)^{2}\left(3 s^{2}-3 s+2\right)\left(\sigma_{1}^{2}-3 \sigma_{2}\right) \sigma_{1} \sigma_{3} \\
& +2(s+1)^{2}\left(-s^{3}+2 s^{2}-4 s+1\right) D_{3,0}(1) \sigma_{3} \\
& +(s+1)^{2}\left(s^{4}+4 s^{3}+8 s^{2}-6 s+5\right) \sigma_{3}^{2}
\end{aligned}
$$

is positive. From (18), $4 \sigma_{2}-\sigma_{1}^{2}$ must be positive. Also, $\sigma_{1}^{2}-3 \sigma_{2}$ is non-negative and $D_{3,0}(1)$ is positive. Therefore, (19) is always positive and (5) holds when $s \leq 0$. In particular,

$$
D_{3,0}(x) D_{3,0}(y)>2 D_{3,0}(x+y) .
$$

Also, $\sigma_{1} \sigma_{2} \geq 9 \sigma_{3}$ gives

$$
D_{3,0}(1)=\sigma_{1} \sigma_{2}-\sigma_{3} \geq 8 \sigma_{3},
$$

which can be written in the useful form

$$
D_{3,0}(x) \geq 8\left(a_{1} a_{2} a_{3}\right)^{x} \quad \text { and } \quad D_{3,0}(y) \geq 8\left(a_{1} a_{2} a_{3}\right)^{y} .
$$

We now use

$$
D_{3, s}(x)=s D_{3,1}(x)+(s-1)^{2} D_{3,0}(x)-s(s-1)(s+3)\left(a_{1} a_{2} a_{3}\right)^{x}
$$

to generate a lengthy expression for

$$
\delta=D_{3, s}(x) D_{3, s}(y)-2(1-s)^{2} D_{3, s}(x+y) .
$$

If $0<s<1$, (3) and (20) give

$$
\begin{aligned}
\delta> & s(2 s-1)(2-s) D_{3,1}(x+y)+s(s-1)^{2}\left[D_{3,1}(x) D_{3,0}(y)+D_{3,0}(x) D_{3,1}(y)\right] \\
& +s^{2}(1-s)(s+3)\left[D_{3,1}(x)\left(a_{1} a_{2} a_{3}\right)^{y}+D_{3,1}(y)\left(a_{1} a_{2} a_{3}\right)^{x}\right] \\
& +s(1-s)^{3}(s+3)\left[D_{3,0}(x)\left(a_{1} a_{2} a_{3}\right)^{y}+D_{3,0}(y)\left(a_{1} a_{2} a_{3}\right)^{x}\right] \\
& +s(s-1)^{2}(s+3)\left(s^{2}+5 s-2\right)\left(a_{1} a_{2} a_{3}\right)^{x+y} .
\end{aligned}
$$

Then (17) and (21) give

$$
\delta>s(2 s-1)(2-s) D_{3,1}(x+y)+s(s-1)^{2}(s+3)\left(s^{2}-11 s+14\right)\left(a_{1} a_{2} a_{3}\right)^{x+y} .
$$

Finally, (16) is used to show that $\delta$ is positive when $0<s<1$.

If $s>1$, and $s \neq 2$ then $D_{3, s}(x)$ can change sign more than once as $x$ increases from 0 to $+\infty$. If $s>1$, (5) cannot hold for all positive $x$ and $y$. But a generalization from (4) and (5) to larger $n$ appears possible. Suppose $a_{1}^{x+y}$, $a_{2}^{x+y}, a_{3}^{x+y}, a_{4}^{x+y}$ are the sides of a quadrangle. If $x$ and $y$ are positive and $s \leq 1$, we conjecture that

$$
D_{4, s}(x) D_{4, s}(y)>4(1-s)^{2} D_{4, s}(x+y)
$$


FinAl Remark. The reader may have some difficulty to see that Theorems 1 , 2,3 , and 5 , are valid when $a_{1}$ is negative. We have the following cases in which the inequalities are reversed. In Theorem 1, $a_{1}=-2, a_{2}=1, n=2, s=-\frac{1}{4}$, $x=1$ and $y=3$. In Theorem 2, $a_{1}=-1, a_{2}=a_{3}=a_{4}=1$. In Theorem 3, $a_{1}<0$, $x=1, y=2$; then let $a_{2} \rightarrow 0$ and $a_{3} \rightarrow 0$. In Theorem 5, $a_{1}=-1, a_{2}=a_{3}=1$, $s=0, x=y=1$.

ACKNOWLedgements. This work was stimulated by correspondence with Professor Dr. H. König, and supported by the Office of Naval Research. The exposition was improved by the labors of a referee, who also suggested a second proof of Lemma 1.

\title{
REFERENCES
}

1. E. F. Beckenbach and R. Bellman, Inequalities, Springer-Verlag, New York, 1965.

2. R. Breusch, American Mathematical Monthly 80, 809, (1973).

3. Lars Gårding, Journal of Mathematics and Mechanics 8, 957, (1959).

4. G. H. Hardy, J. E. Littlewood and G. Pólya, Inequalities, Cambridge University Press, 1952.

5. E. W. Hobson, A Treatise on Plane and Advanced Trigonometry, Dover Publications, New York, 1928.

6. J. L. W. V. Jensen, Acta Mathematica 30, 175, (1905).

7. D. S. Mitrinović, Analytic Inequalities, Springer-Verlag, New York, 1970.

8. A. Pringsheim, Sitzungsberichte der mathematisch-physikalischen Classe der königlichen, Bayerischen Akademie der Wissenschaften zu München 32, 295, (1903).

\author{
C. E. CARroll* \\ DEPARTMENT OF PHYSICS \\ University of Pennsylvania \\ Philadelphia, Pennsylvania 19174 \\ C. C. Yang and S. Ahn† \\ Naval Research Laboratory \\ WASHington, D. C. 20375
}

* Present address: Research School of Chemistry, Australian National University Canberra, A. C. T. 2600 .

$\dagger$ Also at Department of Physics, University of Pennsylvania, Philadelphia, Pennsylvania. 